\title{
¿Qué lugar ocupan actores sociales en el contexto de servicios ecosistémicos? Una revisión en áreas de ecología y biología de la conservación
}

\author{
What role do social actors play in the context of \\ ecosystem services? A review in areas of ecology and \\ conservation biology
}

Que lugar ocupam os atores sociais no contexto dos serviços ecossistêmicos? Uma revisãonasáreas de ecologia e conservação biológica

\author{
Daniela del Castillo \\ Federico di Pasquo ${ }^{b}$ \\ Tomás Emilio Busan ${ }^{c}$ \\ Gabriela Klier $^{d}$ \\ Bettina Mahler
}

aIEGEBA-CONICET- UBA, Facultad de Filosofía y Letras, Grupo de Filosofía de la Biología, Buenos Aires, Argentina. Correo Electrónico: dld.castillo@gmail.com
${ }^{b C}$ ONICET-UBA, Facultad de Filosofía y Letras, Grupo de Filosofía de la Biología, Buenos Aires, Argentina. Correo Electrónico: dipasquof@yahoo.com.ar

'UBA, Facultad de Filosofía y Letras.Grupo de Filosofía de la Biología, Buenos Aires, Argentina. Correo Electrónico: tomasemiliobusan@gmail.com

${ }^{d}$ CONICET-UBA, Facultad de Ciencias Exactas y Naturales, Grupo de Filosofía de la Biología, Buenos Aires, Argentina. Correo Electrónico: gabrielaklier@gmail.com

eIEGEBA-CONICET-UBA, Buenos Aires, Argentina. Correo Electrónico: bemahler@ege.fcen.uba.ar

doi:10.18472/SustDeb.v10n1.2019.19986

\section{RESUMEN}

El enfoque de Servicios Ecosistémicos (SE) constituye una herramienta utilizada para abordar problemáticas ambientales. La valoración de SE en términos ecológicos, monetarios o socioculturales es un elemento clave de esta perspectiva. Realizamos una revisión de artículos científicos centrados en SE publicados entre 2005-2017 en revistas de ecología y biología de la conservación, investigando la forma en quese recuperan voces de diferentes actores sociales (AS). Los resultados indican que predominan las valoraciones ecológica y monetaria por sobre la valoración sociocultural, y que esta última se realiza de una manera que consideramos superficial -sinabordar conflictos entre diferentes AS, y con losinvestigadores seleccionando los SE a valorar. Considerando que los conflictos vinculados con SE están inmersos en contextos sociales complejos, descuidar "dimensiones sociales", puede llevar 
a omitir distancias culturales, diferencias de clase, ubicación geográfica, estilo de vida, y esto puede llevar al desarrollo de opciones de gestión inviables o perjudiciales para los AS involucrados.

Palabras clave: Valoración sociocultural; Dominios de valor; Actores sociales; Revisión de literatura; Servicios ambientales.

\section{RESUMO}

Os serviços ecossistêmicos (SE) são uma ferramenta usada para solucionar problemas ambientais. A avaliação dos SE em termos ecológicos, monetários ou socioculturais é um elemento-chave dessa perspectiva. Realizamos uma revisão de artigos científicos focados em SE publicados entre 2005-2017, investigando a maneira pela qual eles recuperam vozes de diferentes atores sociais (AS). Os resultados indicam que as valorações ecológicas e monetárias predominam sobre a avaliação sociocultural, e que esta última é realizada de uma forma que consideramos superficial - sem abordar os conflitos entre diferentes AS, e com os pesquisadores selecionando os SE a serem avaliados. Considerando que os conflitos relacionados com eles estão inseridos em contextos sociais complexos, que incluem diferentes estilos de vida, negligenciar "dimensões sociais" pode levar à esquecer diferenças de classe, de localização geográfica, de estilo de vida, e isso pode levar ao desenvolvimento de opções de gestão que são inviáveis ou prejudiciais aos AS envolvidos.

Palavras-chave: Valorização sociocultural; Domínios de valor; Atores sociais; Revisão de literatura; Serviços ambientais.

\section{INTRODUÇÃO}

En el contexto de la denominada "crisis ambiental" se ha dado un amplio desarrollo de herramientas que analzan vínculos entre las personas y su ambiente (MEA, 2003; BALVANERA ET AL., 2011; BUSAN Y FOLGUERA, 2018). Entre ellas, los Servicios Ecosistémicos (SE), se transformaron recientemente en una herramienta muy utilizada para abordar problemáticas ambientales (PA)1 dentro del ámbito académico.

Algunos autores (DAILY, 1997) reconocen que la noción de que los ecosistemas brindan servicios a la humanidad surge en la década de 1970 (SCEP, 1970), aunque fue durante la década de 1980 y 1990 que la herramienta fue popularizándose dentro de la academia, hasta que en 1997, después de dos publicaciones muy influyentes (COSTANZA ET AL., 1997; DAILY ET AL., 1997), los artículos enfocados en este concepto aumentaron exponencialmente (FISHER ET AL., 2009).

La publicación de la Evaluación de Ecosistemas del Milenio en 2005 (MEA, 2005) fue otro hito en la historia de los SE;este estudio de 4 años involucró a más de 1300 científicos, y fue encargado por las Naciones Unidas (DEMPSEY Y ROBERTSON, 2012).

El informe, estructurado explícitamente alrededor del concepto de SE como un intento de integrar sustentabilidad ecológica, conservación y bienestar humano, analizó el estado de los SE del mundo y proporcionó recomendaciones para los responsables de políticas ambientales. Después de su publicación, los SE se establecieron firmemente en la agenda de política ambiental internacional (GOMÉZ-BAGGETHUN ET AL., 2010), valorados como una herramienta apropiada para avanzar hacia un manejo sustentable de los ecosistemas (BENNETT ET AL., 2015).Consideramos relevante mencionar el contexto de la política ambiental hacia fines del siglo XX, para entender el éxito de los SE dentro y fuera de la comunidad académica. En la década de 1980, surgió en la política ambiental el discurso de la "Modernización Ecológica” (ME, HAJER, 1995).

Este discurso reconoce el carácter estructural de la PA, asumiendo que instituciones políticas, económicas y sociales existentes deben hacerse cargo del cuidado del ambiente2. Este abordaje descansa sobre la dimensión científico-tecnológica y el conocimiento experto, sin considerar contextos sociales locales ni el carácter situado de las problemáticas consideradas (OLTRA, 2005).

Aunque no existe una única definición de SE, y se mantienen discusiones al respecto (FISHER ET AL., 2009; KULL ET AL., 2015), la mayoría de las investigaciones acepta la siguiente definición: "Ios beneficios 
proporcionados por los ecosistemas a los seres humanos, que contribuyen a hacer que la vida humana sea posible y valga la pena vivirla" (MEA, 2005, p.23).

Dentro de los $\mathrm{SE}$, se reconocen cuatro clases diferentes: servicios de aprovisionamiento, servicios de regulación, que regulan las condiciones en los que los humanos habitan, servicios culturales, que contribuyen a experiencias que benefician directa o indirectamente a las sociedades, y servicios de soporte, que incluyen procesos ecológicos básicos que permiten que se provean los anteriores.

La noción de valor es un elemento central del enfoque de SE. Aquí nos referimos a valor instrumental, reconociéndolo como el valor de una entidad como medio para un fin, que puede ser económico, emocional, etc. Es decir, algo valecuando es considerado valioso por los "valoradores" (KLIER, 2018).

Siguiendo a Gómez-Baggethun y colaboradores (2010), entendemos como valoración una estimación de valor o importancia de algo. La literatura sobre SE destaca la importancia de integrar aspectos sociales, ecológicos y monetarios3 en la valoración de los mismos (DE GROOT ET AL., 2002; FARBER ET AL., 2002), reconociendo la importancia de los tres dominios mencionados. Así, la literatura académica es consistente en llamar a abordajes transdisciplianrios4 de los SE, y reconoce las ventajas que otorga la naturaleza transdisciplinaria del concepto, que abarca mecanismos ecológicos, económicos y sociales, teniendo así la potencialidad de conectar el sistema ambiental con la política y la toma de decisiones (COSTANZA Y KUBISZEWSKI, 2012; SCHROTER ET AL., 2014).

Parece evidente que diferentes actores sociales (AS) tendrán diferentes percepciones, valoraciones y usos de los SE, que podrían derivar en conflictos. Los SE suelen ser presentados, en el marco de la biología de la conservación, como una herramienta prometedora para abordar la PA, porque ofrece la posibilidad de considerar que distintos AS reciben diferentes "beneficios" de los ecosistemas, y que existen interacciones complejas entre el ambiente y entre AS (MEA, 2005;MAASS ET AL., 2005; QUÉTIER ET AL., 2007). Por ejemplo, Gretchen Daily mencionó que "El Marco de SE integra dimensiones biofísicas y sociales de la protección ambiental de una manera que es muy prometedora para abordar la crisis ambiental que probablemente llegará a su máximo en el siglo XXI." (DAILY, 2000, p.333). Por otro lado, Balvanera y colaboradores señalaron que "El concepto de SE además considera el beneficio que distintos actores o sectores de la sociedad reciben de los ecosistemas, así como las complejas interacciones tanto positivas como negativas entre servicios y entre actores o sectores de la sociedad" (BALVANERA et al., 2011, p. 45).

\subsection{OBJETIVOS}

El objetivo de este trabajo es evaluar la forma en que los SE son abordados en la práctica científica, en particular en el área de ecología y biología de la conservación, con especial énfasis en las "dimensiones sociales". Cuando hablamos de "dimensiones sociales" nos referimos a si las investigaciones recuperan las voces de diferentes AS que tienen una relación con los SE analizados, y cómo lo hacen. Más específicamente, nuestro objetivo es reconocer i) cómo los investigadores incluyen las voces de otros AS; ii) qué tan representada está la valoración sociocultural y iii) algunas características de esta valoración.

\section{METODOLOGÍA}

Elegimos revistas que abordan temas ambientales con diferentes enfoques: Conservation Biology y Biological Conservation, dos de las revistas más importantes de Biología de la Conservación; Ecosystem Services, una revista interdisciplinaria que aborda aspectos científicos, políticos y prácticos de los SE; Ecological Economics, una revista transdisciplinaria que integra temas de ecología y economía; Ecology \& Society, una revista interdisciplinaria que se centra en la relación entre la sociedad y los ecosistemas; Journal of Applied Ecology, que se centra en temas de ecología y gestión, cubriendo temas de biología de la conservación.

Más allá de las diferentes perspectivasque presentan las revistas seleccionadas, las mismas tienen en común un perfil orientado hacia la ecología y biología de la conservación. Pese a que los SE son 
abordados desde diferentes enfoques, nos interesa en este artículo centrarnos en revistas vinculadas al área de conservación. El período de tiempo que abordamos fue desde el 2005, momento en que se publicó el MEA, y el 2017 (Apéndice 1).

Seleccionamos artículos que contuvieran las palabras "Ecosystem Services" en el título, y fijamos un límite para analizar 20 artículos por revista. Para Conservation Biology y Biological Conservation, solamente se encontraron 12 y 20 artículos que incluyeran "Ecosystem Services" en el título, respectivamente, por lo que se seleccionaron todos ellos.

En el caso de las demás revistas, como la cantidad de artículos con "Ecosystem Services" en el título superaba el límite fijado, decidimos seleccionar artículos de manera de tener una muestra con representación temporal. Para Ecosystem Services, que comenzó a publicarse en 2012, seleccionamos 3 artículos por año (al azar), analizando así 18 artículos. En el caso de Ecology \& Society, Journal of Applied Ecology y Ecological Economics, seleccionamos en principio 2 artículos por año (al azar) que tuvieran "Ecosystem Services" en el título, y obtuvimos una muestra de 26 artículos por revista.

A continuación, se descartaron artículos que no estaban centrados en SE, quedando así 19, 17 y 24 artículos respectivamente. En el caso de Ecological Economics, como se superaba el límite fijado, se descartaron al azar 4 artículos.

Somos conscientes de que esta selección deja sin cubrir una gran cantidad de literatura científica, sin embargo, consideramos que nuestra muestra es amplia e incluye revistas dentro del área de ecología y biología de la conservación con enfoques diferentes entre sí, lo que nos permite sacar algunas conclusiones para estas áreas. Una vez seleccionados los artículos, respondimos para cada uno de ellos una serie de preguntas vinculadas al modo en que se abordan los SE, con énfasis en cómo se abordan las "dimensiones sociales" (Tabla 1 ).

Tabla 1. Se muestran las diferentes variables analizadas, en torno las cuales se ha organizado el material de trabajo.

\begin{tabular}{|c|c|c|}
\hline Variable & Descripción & Niveles \\
\hline Número de SE analizados & Se contó el número de SE analizados & $1,2,3, \ldots$ \\
\hline Área estudiada & $\begin{array}{l}\text { Se determinó en qué continente fueron } \\
\text { estudiados los SE }\end{array}$ & $\begin{array}{l}\text { A. Del Norte, A. del Sur, A. } \\
\text { central, África, Asia, Oceanía, } \\
\text { Europa, Antártida. }\end{array}$ \\
\hline Tipo de SE analizados & $\begin{array}{l}\text { Se determinó qué tipo de SE fueron } \\
\text { analizados }\end{array}$ & $\begin{array}{c}\text { Aprovisionamiento/ regulación/ } \\
\text { soporte/ cultural }\end{array}$ \\
\hline $\begin{array}{l}\text { Dominios de valoración } \\
\text { considerados }\end{array}$ & $\begin{array}{c}\text { Se determinó que tipo de valoración se } \\
\text { efectuó }\end{array}$ & $\begin{array}{l}\text { Ecológica/ económica/ } \\
\text { sociocultural }\end{array}$ \\
\hline $\begin{array}{l}\text { Participación de actores } \\
\text { sociales locales en la } \\
\text { investigación }\end{array}$ & $\begin{array}{c}\text { Se determinó si los actores locales } \\
\text { participaron en alguna instancia de la } \\
\text { investigación (diseño, implementación, o } \\
\text { análisis) }\end{array}$ & $\mathrm{Si} / \mathrm{No}$ \\
\hline $\begin{array}{l}\text { Objetivos vinculados a } \\
\text { actores sociales }\end{array}$ & $\begin{array}{l}\text { Se determinó, a partir de los objetivos, si las } \\
\text { investigaciones estaban centradas en AS }\end{array}$ & $\mathrm{Si} / \mathrm{No}$ \\
\hline $\begin{array}{l}\text { Abordaje de conflictos } \\
\text { entre actores sociales }\end{array}$ & $\begin{array}{l}\text { Se determinó si las investigaciones } \\
\text { consideraban más de un grupo de AS, y si } \\
\text { abordaban conflictos entre ellos }\end{array}$ & $\mathrm{Si} / \mathrm{No}$ \\
\hline $\begin{array}{l}\text { Selección de SE a } \\
\text { valorar en la valoración } \\
\text { sociocultural }\end{array}$ & Se determinó cómo se elegían los SE a valorar & $\begin{array}{l}\text { Unilateralmente (investigadores) } \\
\text { /en conjunto con actores locales }\end{array}$ \\
\hline $\begin{array}{l}\text { Presentación de la } \\
\text { valoración sociocultural } \\
\text { en forma de lista de } \\
\text { prioridades }\end{array}$ & $\begin{array}{l}\text { Se determinó cómo se expresó el resultado } \\
\text { de la valoración sociocultural }\end{array}$ & Lista/Otras \\
\hline
\end{tabular}


Fuente: Autores.

\title{
3 RESULTADOS
}

\section{Número de SE analizados}

Encontramos que más de la mitad de los artículos estudiaron 6 SE o menos, con un pico de $15 \%$ de trabajos estudiando un único SE (Figura 1).

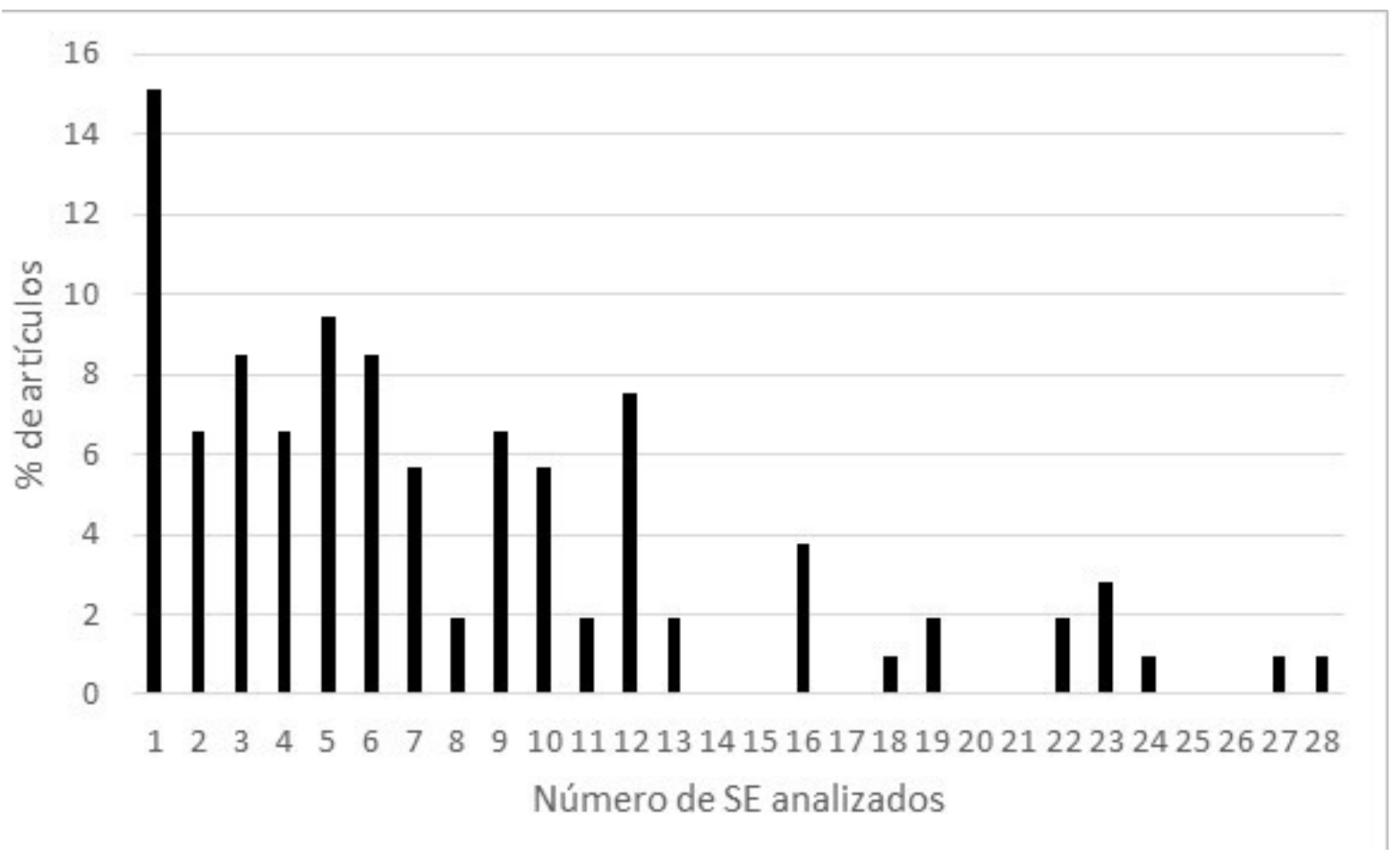

Figura 1. Porcentaje de artículos analizados que estudian una determinada cantidad de SE.

\author{
Fuente: Autores.
}

\section{Área estudiada}

La mayoría de los artículos analizados investigó SE en Europa (44\%) y América del Norte (18\%). Antártida, Amércia del Sur y Central fueron los continentes menos estudiados (0\%,6\% y $3 \%$ respectivamente), mientras que África, Asia y Oceanía presentaron valores intermedios (11\%, 9\% y $9 \%$ respectivamente).

\section{Tipos de SE analizados}

El $81 \%$ de los artículos estudió servicios de regulación, mientras que el $67 \%$ y $65 \%$ estudiaron servicios de aprovisionamiento y culturales, respectivamente. Solo el $22 \%$ estudió servicios de soporte (Figura 2). 


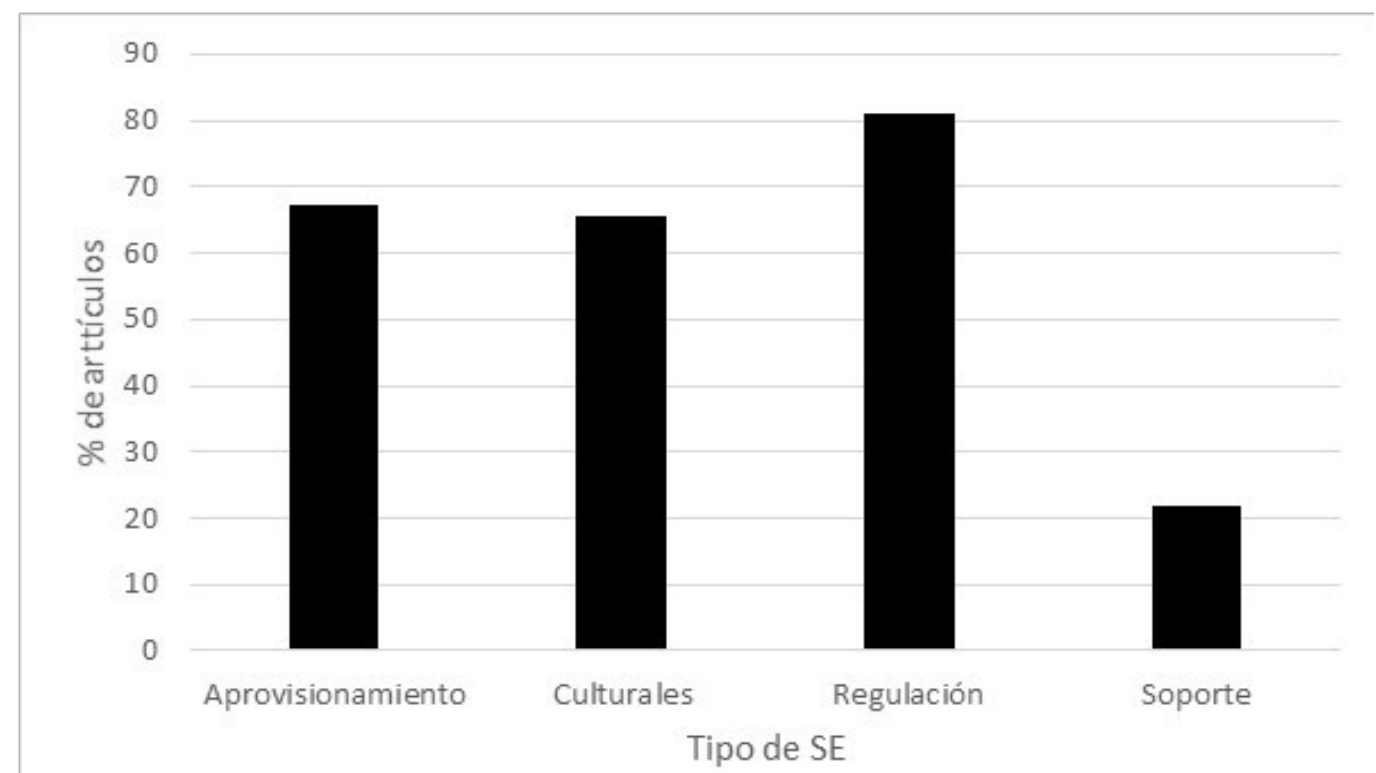

Figura 2. Porcentaje de artículos que estudian cada tipo de SE.

Fuente: Autores.

Por otro lado, solo el 10\% estudió los 4 tipos de SE simultáneamente. El 31\% de los trabajos abordó SE de aprovisionamiento, regulación y culturales, y el $25 \%$ de los trabajos investigó un único tipo de SE (1\% soporte, $12 \%$ regulación, $7 \%$ culturales, $5 \%$ aprovisionamiento).

\section{Dominios de valoración considerados}

La valoración ecológica fue utilizada en el $42 \%$ de los artículos, la monetaria en el $41 \%$, y la sociocultural en el $29 \%$ (Figura 3). Por otro lado, la gran mayoría de los trabajos incluyó un único dominio de valoración (89\%), el $8 \%$ de los trabajos utilizó simultáneamente valoración ecológica y monetaria, el $1 \%$ utilizó valoraciones ecológicas y socioculturales, monetarias y socioculturales, y, de manera similar solo el $1 \%$ utilizó los tres dominios de valoración (Figura 4).

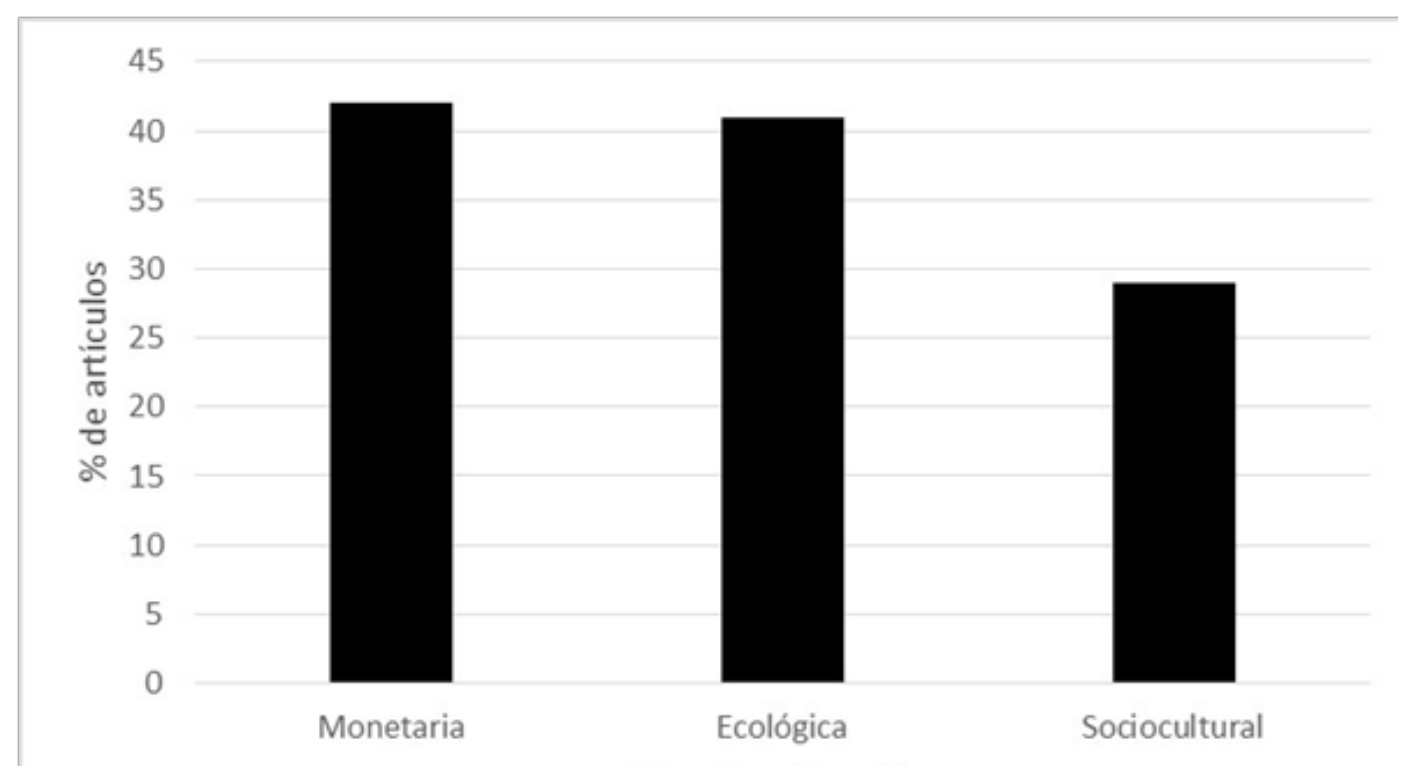

Tipo de valoración

Figura 3. Porcentaje de artículos que utilizan los tres dominios de valoración de SE.

Fuente: Autores. 


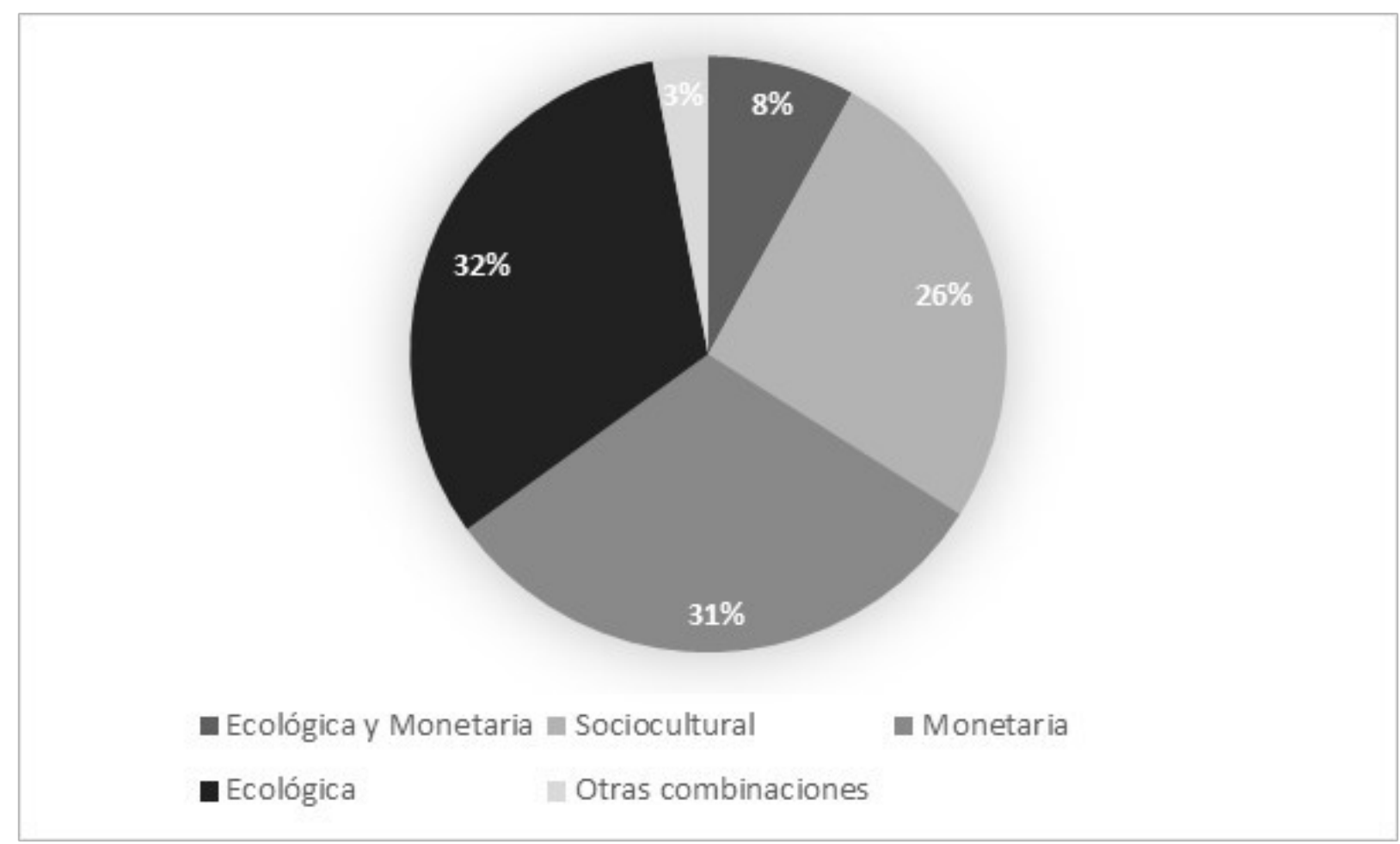

Figura 4. Porcentaje de artículos que utilizan los tres dominios de valoración. Se muestra el porcentaje de artículos que incluye dos o más dominios.

Fuente: Autores.

\section{Participación de actores locales en la investigación}

En el $61 \%$ de los artículos, los AS locales no estuvieron incluidos en ninguna instancia de la investigación (Figura 5).

\section{Objetivos vinculados a actores sociales}

El análisis de los objetivos mostró que en más del 70\% de los artículos, los AS no fueron parte de los objetivos de la investigación (Figura 5).

\section{Abordaje de conflictos entre actores sociales}

Solo una pequeña parte de los estudios (13\%) abordó conflictos entre AS (Figura 5), lo que significa que más de la mitad de los estudios que utilizan la valoración sociocultural, consideran un único grupo de AS al valorar los SE, o consideran más de un grupo de AS, pero estos se homogeneizan por la forma en que se realizan las investigaciones. Es decir, en este último caso, los conflictos entre diferentes AS serían invisibilizados implícita o explícitamente.

\section{Selección de SE a valorar}

En el $58 \%$ de los artículos, los investigadores identificaron unilateralmente los SE a ser valorados, mientras que en el $42 \%$ de los artículos, los SE seleccionados para su valoración surgieron a partir de interacciones entre investigadores y AS en contacto con los ecosistemas estudiados (Figura 5).

\section{Presentación de valoración sociocultural}

En el $75 \%$ de los artículos analizados, la valoración sociocultural se presentó como una lista de prioridades (es decir, qué SE es preferido sobre otro, figura 5). 


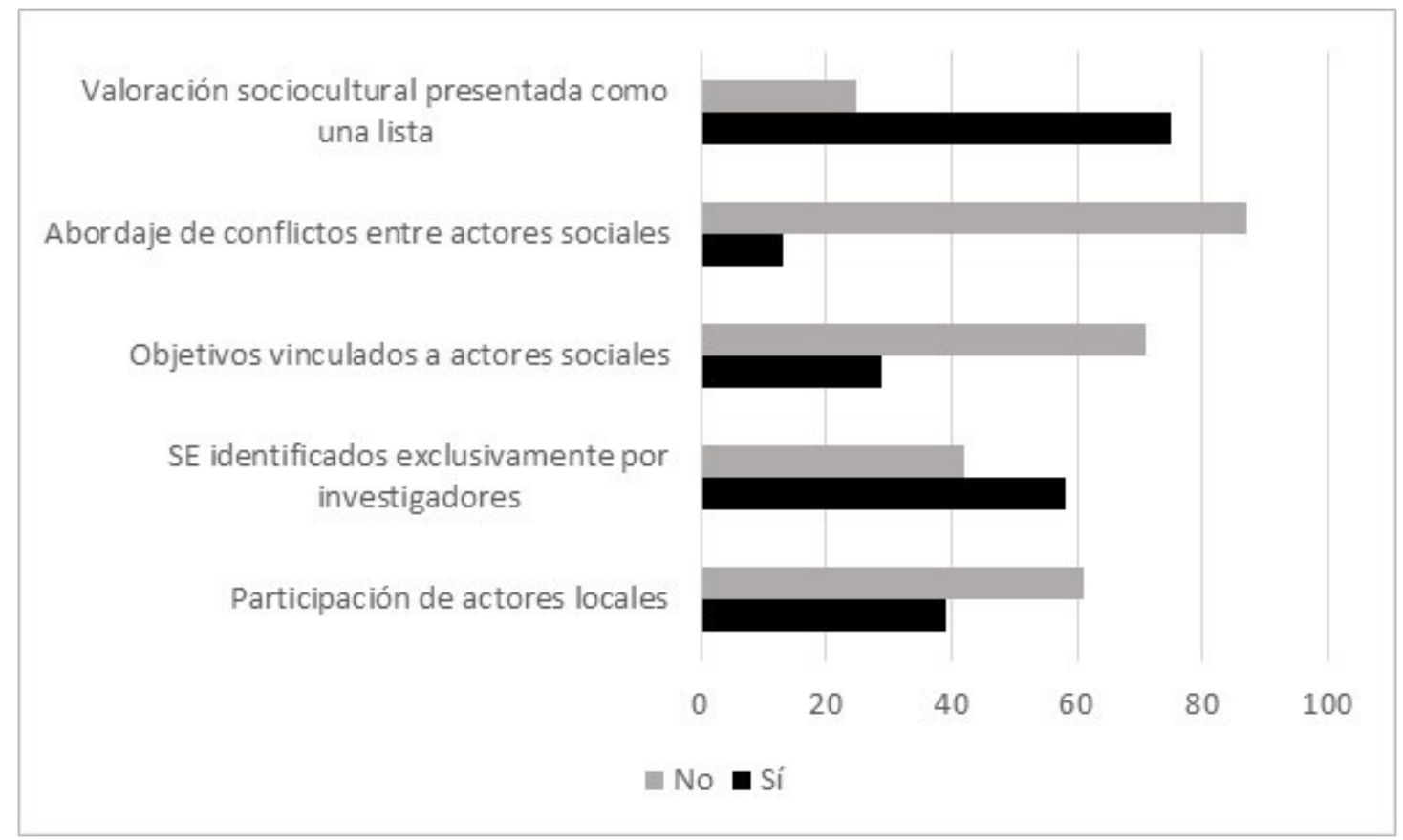

Figura 5. Porcentaje de artículos que abordan "dimensiones sociales". Fuente: Autores.

\section{DISCUSIÓN}

La mayoría de las investigaciones analizadas estudió 6 SE o menos, con un máximo de 15\% de los trabajos que incluyeron un único SE. Este número resulta bajo si se lo compara con la cantidad de SE descriptos en la literatura. Por ejemplo, en el reconocido artículo de Costanza y colaboradores (1997) se describen 17 SE, y en otro estudio muy citado (HEIN ET AL., 2006) se describen 25 SE.

En revisiones anteriores, Seppelt y colaboradores (2011) encontraron que aproximadamente el 50\% de los estudios a nivel global consideraron 5 SE o menos, mientras que Nieto Romero y coaboradores (2014), encontraron que más del $60 \%$ de los estudios centrados en agroecosistemas mediterráneos evaluaron un único SE.

En relación a esto, el enfoque de SE surge de la conceptualización de los ecosistemas como conjuntos de sistemas integrados (LATERRA ET AL., 2011), pero al estudiar unos pocos SE, no parece posible abordar los ecosistemas de manera integral. Compartimentar el ambiente presenta dificultades técnicas (entre otras), ya que enmascara el hecho de que cada función de un ecosistema se relaciona y depende de las demás (VATN, 2000; KOSOY Y COBRERA, 2010). El estudio de un único (o unos pocos) SE, no permite ver como cada servicio se relaciona con el resto del ecosistema.Así, la práctica científica parece dejar de lado una ventaja que ofrece la perspectiva SE.

En cuanto a la distribución geográfica de los SE analizados,el 62\% de los artículos investigó SE en América del Norte y Europa. Esto resulta esperable, dado que las revistas analizadas son europeas y norteamericanas, por lo que es lógico que haya un sesgo en ese sentido.

Sin embargo, existe un sesgo vinculado a la relevancia de las revistas europeas y norteamericanas dentro de las áreas de biología de la conservación y ecología. En este sentido, en un ranking de relevancia de SClmago (http://www.scimagojr.com), dentro de las 10 revistas actuales más relevantes de ecología, cinco resultan europeas, dos norteamericanas, dos asiáticas y una sudamericana, y resultados similares se presentan en revistas de conservación de la naturaleza.

En relación al tipo de SE abordados, los SE de regulación fueron los más estudiados, seguidos por los de aprovisionamiento, culturales y de soporte en ese orden, con una sub-representación de los 
servicios de soporte. De manera similar, Vihervaara y colaboradores (2010), encontraron que los SE de aprovisionamiento y regulación fueron los más estudiados en todo el mundo, mientras que Nieto Romero y colaboradores (2014) informó que la investigación sobre SE en agroecosistemas mediterráneos se centró principalmente en SE de aprovisionamiento.

Cabe aquí mencionar que existe una discusión teórica sobre los SE de soporte, que son necesarios para que se den los restantes y por lo tanto la valoración de todos ellos necesariamente implica su incorporación, aunque sea de manera indirecta. Por eso, estos servicios suelen obviarse debido los problemas de doble conteo asociados (FISHER ET AL. TURNER, 2008). Por otro lado, algunos autores mencionan que los servicios de soporte se diferencian de todos los demás ya que su utilidad para las personas es indirecta, son poco tangibles y de largo plazo, por lo que existe poca conciencia de su importancia (ABSON Y TERMANSEN, 2011; CONABIO, 2016). Esta sub-representación de los SE de soporte podría entonces estar relacionada con que la investigación sobre SE está dirigida principalmente anecesidades humanas a corto plazo. Así, variables que cambian más lentamente, son usualmente ignoradas en la investigación así como en la subsiguiente aplicación de políticas ambientales, haciendo peligrar seriamente la existencia de todos los servicios en el largo plazo (considerando que los servicios de soporte son necesarios para la provisión de los demás).

Aquí vale la pena reflexionar el concepto de SE como una herramienta para abordar PA, y qué consecuencias pueden asociarse con el hecho de que las investigaciones estudien generalmente los beneficios directos provistos por los ecosistemas, otorgando un lugar menor a los servicios de soporte.

En cuanto a los dominios de valoración, la mayoría de los trabajos incluyó un único dominio, y solo el $1 \%$ consideró simultáneamente los tres. Esta tendencia coincide con aquella hallada por Nieto Romero y colaboradores (2014) en agroecosistemas mediterráneos. Así, aunque los SE suelenpresentarse como una herramienta prometedora para abordar PA porque ofrecen la posibilidad de integrar dimensiones sociales y ecológicas (DAILY, 2000; BALVANERA ET AL., 2011), nuestros resultados sugieren que los estudios de caso no explotan esta posibilidad, ya que usualmente se enfocan en un único dominio de valor.

Martin López y colaboradores (2014), mostraron que los diferentes métodos de valoración (ecológico, monetario y sociocultural) utilizados para valorar SE revelan información diferente. Según el autor, los métodos de valoración pueden concebirse como instituciones de articulación de valores, como un marco que influye en los valores que se presentan.

En la misma línea, Vatn (2005) define las instituciones de articulación de valores como un conjunto de reglas construidas que especifica las condiciones bajo las cuales se expresarán los valores, tales como i) qué tipo de datos se considerarán relevantes (p.e., la valoración monetaria se hace en términos monetarios), ii ) quién participa en la valoración (p.e., la valoración monetaria y ecológica es efectuada por expertos) y iii) en carácter de qué (p. e., la valoración sociocultural considera a las personas como beneficiarias de los SE). Así, si la selección del dominio de valor determina el resultado, entonces, los autores afirman que la investigación sobre SE debe combinar dominios de valor diferentes para presentar una visión más amplia de los servicios que abordan.

Además, es importante que los autores cuya investigación se centra en un cierto dominio de valor expliquen su elección (qué consideran valioso, por qué y para quién lo consideran valioso). Por otro lado, aunque la valoración sociocultural fue menos utilizada que las demás, esta diferencia no resultó tan grande como en revisiones anteriores (p.e. VIHERVAARA ET AL., 2010; NIETO-ROMERO ET AL., 2014).

Este aumento puede estar vinculado con el aumento del reconocimiento del aporte que pueden hacer las ciencias sociales en los conflictos ambientales que se dio en los últimos años (SANDBROOK ET AL., 2013), donde varios autores han comenzado a destacar la importancia de considerar intereses y preocupaciones de AS locales para conseguir éxito en proyectos de conservación. Pese a esto, las valoraciones monetarias y ecológicasaún predominan en la literatura. Presentamos algunas consideraciones al respecto, sin pretender ser exhaustivos, mencionando cuestiones que consideramos relevantes.

En cuanto a la valoración monetaria de los SE, la idea que subyace la misma, es que "Si asignamos precio al ambiente correctamente (...) entonces las personas y el ambiente salen ganando. Si la 
naturaleza puede ser abstraída racionalmente y puede asignársele un precio (...) entonces el riesgo y la degradación ambiental pueden ser medidos (...) y generalmente, minimizados" (Sullivan, 2009, p.256).

La autora menciona que valorar no equivale a asignar un precio, y que asignar un valor monetario al ambiente no implica que vayamos a incorporar apreciación o afecto hacia el mundo no humano. Por otro lado, Gómez-Baggethun y colaboradores (2010) mencionan que por razones éticas a algunas cosas no debería asignárseles un precio5. Desde un punto de vista práctico, las investigaciones que aplican la valoración monetaria de $\mathrm{SE}$, suelen establecer unidades discretas de $\mathrm{SE}$, que puedan incorporarse a ecuaciones económicas.

Así, el intento de compartimentar los SE, está en desacuerdo con el hecho de que las funciones de los ecosistemas están inevitablemente vinculadas entre sí (VATN, 2000). Respecto de la valoración ecológica, suele usarse la palabra valor entendiéndolo como una medición numérica. Siguiendo a Gómez-Baggethun y colaboradores (2014), las medidas ecológicas (p. e., metros cúbicos de madera) representan cuentas ambientales, pero no se califican automáticamente como valores. Estas medidas califican como valores cuando se asocian con alguna atribución de importancia social. Sin embargo, esta relación no suele explicitarse, oscureciendo el por qué, para qué, y para quién son importantes los SE valorados.

En relación a alcances y límites del trabajo, cabe destacar el perfil de las fuentes analizadas. Hemos abordado revistas con un perfil de ecología y biología de la conservación. Sin embargo, el enfoque de SE no es exclusivo de estas áreas, sino que es retomado también por investigaciones de corte más social.

En este sentido, vale reconocer que existen artículos que escapan al área abordada por nosotros, que plantean roles centrales para los AS locales (DEDEURWAERDERE ET AL.,2016; STENSEKE YLARIGAUDERIE, 2018). Más aún, dentro de las áreas que analizamos, hay ciertos artículos que dan lugares centrales a Ios AS locales (BALVANERA Y COTLER 2009, CÁCERES ET AL., 2015.). Pese a esto, nuestra revisión apunta a determinar aspectos generales vinculados al rol que se da a los AS dentro de las investigaciones en el área de ecología y conservación.

Volviendo a nuestros objetivosy en relación con los ejes analizados, resaltamos resultados relacionados con "dimensiones sociales" en revisiones anteriores: diferentes autores han informado que la mayoría de los estudios de SE no involucran a AS locales en la valoración de los mismos (SEPPELT ET AL., 2011; NIETOROMERO ET AL., 2014). Seppelt y colaboradores (2011), encontraron que menos del $40 \%$ de los estudios sobre SE informaron algún grado de participación de AS locales, concluyendo que la selección de temas a estudiar suele estar impulsada por la curiosidad, sin responder a problemas locales específicos.

En la mayoría de los artículos que analizamos los AS locales no se incluyeron en los objetivos de las investigaciones ni participaron en ninguna instancia de la investigación. Los artículos raramente consideraron a diferentes AS y abordaron los conflictos entre ellos. Esto significa que incluso cuando las investigaciones incluyeron valoración sociocultural, por lo general se centraron en un solo grupo de AS, sin considerar la relación de diferentes AS con los SE, ni posibles vinculados a diferentes percepciones, usos y valoraciones de los mismos.

Considerando que los conflictos vinculados a SE están inmersos en contextos sociales complejos, con AS que presentan diferentes visiones y formas de relacionarse con el mundo (y con los SE), descuidar estos aspectos puede llevar a dejar de lado distancias culturales, diferencias de clase, sexo, ubicación geográfica, estilo de vida en general (KLIER, 2018), y esto no solo presenta una versión incompleta del conflicto, sino que puede conducir al desarrollo de opciones de gestión inviables e incluso perjudiciales paraAs locales.

Varios autores reconocen que las intervenciones de conservación requieren cambios en el comportamiento de las personas para tener éxito, por lo que los enfoques socioculturales deben considerarse claves para las evaluaciones de los SE (MENZEL Y TENG, 2010; CHAN ET AL., 2012; MARTINLOPEZ ET AL., 2014).

Por otro lado, Berkes (2004) considera lasPA como problemas que no pueden separarse de cuestiones de valores, equidad y justicia social, y menciona que en estos conflictos la figura de un "experto objetivo y 
desinteresado" no tiene sentido. Por el contrario, aquí los investigadores y las partes interesadas deben trabajar juntos para definir preguntas y objetivos. La participación de AS locales en la investigación es importante, pero no porque sean ellos quienes deben aplicar las medidas de conservación impuestas por agentes externos de una manera verticalista, sino porque son ellos quienes deben definir, en diálogo con investigadores y legisladores, conflictos, objetivos y medidas de conservación (COLCHESTER, 2000).

Teniendo esto en cuenta, es esencial abordar las PAsin dejar de lado qué AS están involucrados, quiénes y cómo se ven afectados por las políticas y prácticas propuestas para mejorar los SE y el bienestar "humano". Sin embargo, nuestros resultados apuntan a que, pese al creciente reconocimiento de las contribuciones de las ciencias sociales en la conservación y del aumento de la valoración sociocultural, las "dimensiones sociales" no son un tema central en la investigación de SE. La cantidad de artículos que utilizan la valoración sociocultural por sí misma no es un buen indicador de cómo se toman en cuenta las "dimensiones sociales"; aunque la valoración sociocultural es llevada a cabo por los AS locales, en general son los investigadores quienes deciden los SE a valorar.

Es decir, aunque los AS expresan cómo valoran los SE, éstos son determinados por los investigadores. Además, la valoración generalmente se presenta como una lista de SE ordenados según su prioridad. Así, el valor se "traduce" a una lista de prioridades, el valor queda expresado como una preferencia: qué SE se prefiere sobre otro. Nos preguntamos, entonces, cómo esta lista está vinculada a la importancia que las personas le dan a los SE en su vida. Es decir, hasta qué punto un orden jerárquico representa la importancia que los SE tienen para las personas. Aunque en las evaluaciones socioculturales reconocemos intenciones de incluir otras voces dentro de la investigación, y los AS locales sonlos que asignan valor, lo hacen bajo la perspectiva de $\mathrm{SE}$, un marco particular que presenta sus propias suposiciones. Nos interesa entonces preguntar qué se deja de lado al abordar la relación entre personas y ecosistemas desde la perspectiva de SE.

El dominio actual de los SE para la gestión ambiental -donde aparece como un concepto valorativamente neutral y epistémicamente objetivo- oculta el hecho de que hay decisiones implícitas en su encuadre y en su aplicación (KULL ET AL., 2015). Los SE están inmersos en sistemas de significados, narrativas y creencias (HIRSCH ET AL., 2011), sujetos a suposiciones y formas particulares de entender el mundo.

Siguiendo a Sullivan (2009), la perspectiva de SE considera que los ecosistemas proporcionan "servicios" a la humanidad: "la naturaleza" sirve a "los humanos". Además, las personas que viven en paisajes valorados por su provisión de SE se construyen como servidores, tal como lo perciben los expertos en políticas y técnicos.La perspectiva de SE enfatiza las relaciones amo-servidor entre los reinos humanos y no humanos, extendiéndose aún más entre aquellos "expertos en" y "habitantes de" paisajes valorados (usualmente en términos monetarios o ecológicos) por su provisión de SE.

Para concluir, Gómez-Baggethun y De Groot (2007) mencionan que "naturaleza", "ecosistemas" y "capital natural" son conceptos que pertenecen respectivamente al lenguaje convencional, a la ecología y la economía, y que el uso de uno u otro término será apropiado dependiendo del contexto. Siguiendo di Pasquo y colaboradores (2018), consideramos importante recordar que la naturaleza no es el ecosistema ni el capital natural, y que el papel hegemónico de la ecología y la economía puede ser problemático ya que monopolizan el significado que tenemos de ella. Nos preguntamos si es posible que concebir la naturaleza como un proveedor de servicios para la humanidad, pueda abrir caminos que conduzcan a su apreciación y cuidado por parte de las personas.

\section{CONCLUSIÓN}

A partir del recorrido trazado, delineamos lasconclusiones que consideramos más relevantes: a) Se estudian pocos $\mathrm{SE}$, y se abordancon más frecuencia servicios de aprovisionamiento y regulación, y con menosfrecuencia aquellos de soporte; b) predominan las valoraciones ecológica y monetaria por sobre la valoración sociocultural; c) la valoración sociocultural se realiza de una manera que consideramos superficial. Resulta vital acompañar estos resultados con una reflexión profunda sobre los SE. 
Así, enmarcando a los mismos dentro de una perspectiva más amplia, volvemos a la ME, reconociéndola como uno de los discursos hegemónicos6actuales (MARCH, 2013). Este discurso supone que el crecimiento económico puede alcanzarse a través de la comodificación de recursos, avances en desarrollo científico y tecnológico e instituciones de mercado, prometiendo crecimiento económico ligado a menores impactos ambientales y desarrollo sustentable donde "todos" ("humanidad" y ambiente) pueden ganar.

Sin embargo, estas promesas y los supuestos en que se basan, han dado lugar a la apertura de nuevas esferas de circulación y acumulación de capital (p.e. circuitos de pago por SE). La ME no cuestiona conceptos como desarrollo y progreso, ni los patrones de producción y consumo que han provocado la actual crisis ambiental, a la vez que no tiene en cuenta a todos aquellos marginados por el modelo socioeconómico de desarrollo predominante (MARTINEZ ALIER, 2009; BAKARI, 2014).

En este sentido, nos sentimos escépticos hacia herramientas surgidas desde este discurso y en particular hacia los SE, ya que terminan por legitimar el estado actual de las cosas, y esto se mantendría aún en un escenario donde la investigación diera un lugar menos degradado a AS locales.

Consideramos que la PA no puede solucionarse simplemente reconociendo SE relevantes y estableciendo métodos adecuados para su valoración. El enfoque de SE podría ser una herramienta valiosa para abordar la PA, siempre que sea enmarcada en una discusión profunda acerca de nuestro estilo de vida y de los patrones actuales de producción y consumo.

Dado que el enfoque de SE no problematiza ninguno de los aspectos mencionados, se corre el riesgo de creer que la PA puede ser reducida a un mero equilibrar el uso de cada SE. Así, creemos que es fundamental mantener abiertos interrogantes en torno al discurso que subyace a los SE-la ME- sin darlo por sentado, y repensar propuestas alternativas, como la "justicia ambiental" (LEFF, 2001; MARTínEZ ALIER, 2009) o el "decrecimiento económico" (MARTínEZ ALIER, 2011), que ya no buscan conciliar lo irreconciliable: el crecimiento económico y el cuidado ambiental.

\section{NOTAS}

${ }^{1}$ Entendemos la problemática ambiental como la transformación acelerada de los ecosistemas del mundo por mano humana, que estaría conduciendo a un colapso del planeta en el mediano plazo (adaptado de MEA, 2005).

${ }^{2}$ La noción de ambiente designa la fauna, la flora, los lagos, etc. Esto incluye también elementos intervenidos culturalmente que ya estaban antes de dicha intervención humana (por ejemplo: un lago intervenido) (MONARES, 1999; GUDYNAS, 2015)

${ }^{3}$ Entendemos la valoración monetaria como aquella que utiliza el dinero como unidad de medida, la valoración ecológica como aquella que mide magnitudes de alguna propiedad biofísica (p. e. toneladas de carbono secuestrado) y la valoración sociocultural como aquella en las que AS asignan valor en términos no monetarios (para una discusión sobre métodos de valoración, verGÓMEZ-BAGGETHUN ET AL., 2014).

${ }^{4}$ Existen diferentes términos que dan cuenta del grado de integración disciplinar. Multidisciplinariedad: la asociación entre disciplinas con poco intercambio, aportando a un problema común. Interdisciplinariedad: sintetiza nexos entre las disciplinas para generar un "todo" coherente, con una fuerte cooperación entre áreas científicas (REYERS ET. AL., 2010). Transdisciplinariedad: integra áreas científicas y otras áreas de conocimiento (REYERS ET AL. 2010). Aquí se difuminan los límites entre el conocimiento científico y los conocimientos particulares de cada sociedad en un momento y lugar (HORLICK-JONES Y SIME 2004).

${ }^{5}$ Esta discusión es indudablemente esencial, y está directamente relacionada con los SE, pero está más allá del alcance de este trabajo abordarla en profundidad; para una discusión sobre los problemas de la mercantilización de la naturaleza, ver Sullivan, 2013.

6 Entendemos pensamiento hegemónico como aquel que se asienta como "lo dado", siempre mediado por relaciones de poder (GRAMSCI, 1975). 


\section{BIBLIOGRAFÍA}

ABSON, D. J.; TERMANSEN, M. Valuing ecosystem services in terms of ecological risks and returns. Conservation Biology, v. 25, p. 250-258, 2011.

BAKARI, M. E. K. Sustainability's inner conflicts: from 'ecologism'to 'ecological modernization'. Journal of Sustainable Development Studies, v. 6, p. 1-28, 2014. Disponible en: http://www.infinitypress.info/index.php/ jsds/article/view/618

BALVANERA P., et al. Marcos conceptuales interdisciplinarios para el estudio de los servicios ecosistémicos en América Latina, p. 39-67. In: INTA Eds. El valor ecológico, social y económico de los servicios ecosistémicos. Conceptos, herramientas y estudio de casos, Buenos Aires, 2011. Disponible en: https://ced.agro.uba.ar/ubatic/ sites/default/files/files/libro_serv_ecosist/pdf/Capitulo_02.pdf

BALVANERA, P. y COTLER, H (responsables). Estado y tendencias de los servicios ecosistémicos. En: CONABIO (ed.). Capital natural de México, vol. II: Estado de conservación y tendencias de cambio México: Comisión Nacional para el Conocimiento y Uso de la Biodiversidad, 2009, p. 185-245.

BENNETT, E.; CRAMER, W.; BEGOSSI, A.; CUNDILL, G.; DÍAZ, S.; EGOH, B; LEBEL, L.Linking biodiversity, ecosystem services, and human well-being: three challenges for designing research for sustainability. Current Opinion in Environmental Sustainability, v. 14, p.76-85, 2015.

BUSAN, T.; FOLGUERA, G. Estudio desde la Filosofía de la Ecología de la noción de impacto ambiental: relación entre los saberes que la conforman y sus consecuencias epistémicas. Prometeica,v.16, p. 43-56, 2018. Disponible en: https://doi.org/10.24316/prometeica.v0i16.209

CÁCERES, D. M.; TAPELLA, E.; QUÉTIER, F.; DÍAZ, S. The social value of biodiversity and ecosystem services from the perspectives of different social actors. Ecology and Society, v. 20, p. 62-81, 2015. Disponible en: https://ri.conicet. gov.ar/bitstream/handle/11336/22497/CONICET_Digital_Nro.85402f34-41f8-446c-a897-d3dc3f17cc03_A. pdf?sequence $=2 \&$ isAllowed $=y$

CHAN, K. M.; SATTERFIELD, T.; GOLDSTEIN, J. Rethinking ecosystem services to better address and navigate cultural values. Ecological economics,v. 74, p. 8-18, 2012.

COLCHESTER, M. Self-Determination or Environmental Determinism for Indigenous Peoples in Tropical Forest Conservation. Conservation Biology,v. 14, p. 1365-1367, 2000.

Comisión Nacional para el Conocimiento y Uso de la Biodiversidad (CONABIO) y Secretaría del Medio Ambiente del Distrito Federal. La biodiversidad en la Ciudad de México. México, 2016. Disponible en: https://www. biodiversidad.gob.mx/region/EEB/pdf/Volumen\%201-cdmx_web.pdf

COSTANZA, R.; KUBISZEWSKI, I. The authorship structure of "ecosystem services" as a transdisciplinary field of scholarship. Ecosystem Services,v. 1, p.16-25, 2012.

COSTANZA, R.et al. The value of the world's ecosystem services and natural capital. Nature, v. 387, p. 253-260, 1997.

DAILY, G. C. Management objectives for the protection of ecosystem services. Environmental Science \& Policy,v. 3, p. 333-339, 2000.

DAILY, G. C. Nature's Services: Societal Dependence on Natural Ecosystems.Washington DC: Island Press, 1997.

DEDEURWAERDERE, T.; ADMIRAAL, J.; BERINGER, A.; BONAIUTO, F.; CICERO, L.,; FERNANDEZ-WULFF, P.;\& MELINDI-GHIDI, P. Combining internal and external motivations in multi-actor governance arrangements for biodiversity and ecosystem services. Environmental Science \& Policy, v. 58, p. 1-10, 2016.

DEMPSEY, J.; ROBERTSON, M. M. Ecosystem services: Tensions, impurities, and points of engagement within neoliberalism. Progress in Human Geography, v. 36,p. 758-779, 2012. Disponible en: https://www.univie.ac.at/ intpol/website2014/wp-content/uploads/2015/11/Depsey_Robertson_2012_Ecosystem-services.pdf 
DE GROOT, R. S.; WILSON, M. A.; BOUMANS, R. M. A typology for the classification, description and valuation of ecosystem functions, goods and services. Ecological economics, v. 41, p. 393-408, 2002.

DI PASQUO, F.; BUSAN, T. E.; KLIER, G.; DEL CASTILLO, D. Valores, ecología de ecosistemas y problemática ambiental. Ponencia: XI Encuentro AFHIC. Asociación de Filosofía e Historia de la Ciencia del Cono Sur. Buenos Aires, Argentina, 2018.

FARBER, S. C.; COSTANZA, R.; WILSON, M. A. Economic and ecological concepts for valuing ecosystem services. Ecological economics,v. 41, p. 375-392, 2002.

FISHER, B.; TURNER, R. K.; MORLING, P. Defining and classifying ecosystem services for decision making. Ecological economics, v. 68, p. 643-653, 2009.

GÓMEZ-BAGGETHUN, E. et al. State-of-the-art report on integrated valuation of ecosystem services. EU FP7 OpenNESS Project Deliverable, v. 4, p.1-33, 2014. Disponible en: http://www.openness-project.eu/sites/default/ files/Deliverable\%204\%201_Integrated-Valuation-Of-Ecosystem-Services.pdf

GÓMEZ-BAGGETHUN, E.; DE GROOT, R.; LOMAS, P. L.; MONTES, C. The history of ecosystem services in economic theory and practice: from early notions to markets and payment schemes. Ecological economics, v. 69, p. 1209 1218, 2010.

GÓMEZ-BAGGETHUN, E.; DE GROOT, R. Capital natural y funciones de los ecosistemas: explorando las bases ecológicas de la economía. Revista Ecosistemas,v. 16, p. 4-14, 2007. Disponible en: http://www.revistaecosistemas. net/articulo.asp?Id=496

GRAMSCI, A. El materialismo histórico y la filosofía de Benedetto Croce. México D.F.: Juan Pablos, 1975.

GUDYNAS, E. Derechos de la Naturaleza. Buenos Aires: Tinta Limón, 2015.

HEIN, L.; VAN KOPPEN, K.; DE GROOT, R. S.; VAN IERLAND, E. C. Spatial scales, stakeholders and the valuation of ecosystem services. Ecological economics,v. 57, p. 209-228, 2006.

HIRSCH, P. D.; ADAMS, W. M; BROSIUS, J. P.; ZIA, A.; BARIOLA, N.; DAMMERT, J. L. Acknowledging conservation trade-offs and embracing complexity. Conservation Biology, v. 25, p. 259-264, 2011.

HORLICK-JONES, T., Sime, J. Living on the border: knowledge, risk and transdisciplinarity. Futures, v. 36, p. 441456, 2004.

KLIER, G. Tiempos modernos: un análisis sobre los discursos de la Biología de la Conservación. 209 p. Tesis Doctoral. Universidad de Buenos Aires. Buenos Aires, Argentina, 2018.

KOSOY, N.; CORBERA, E. Payments for ecosystem services as commodity fetishism. Ecological economics,v. 69, p. 1228-1236, 2010.

KULL, C. A.; DE SARTRE, X. A.; CASTRO-LARRAÑAGA, M. The political ecology of ecosystem services. Geoforum, v. 61, p. 122-134, 2015.

LATERRA, P.; JOBBÁGY, E. G.; PARUELO, J. M. Valoración de servicios ecosistémicos: Conceptos, herramientas y aplicaciones para el ordenamiento territorial. Ediciones INTA, Buenos Aires, Argentina, 2011. Disponible en: https://inta.gob.ar/sites/default/files/script-tmp-inta_valoracion_de_servicios_ecosistemicos.pdf

LEFF, E. Justicia ambiental: Construcción y defensa de los nuevos derechos ambientales culturales y colectivos en América Latina. Programa de las Naciones Unidas para el Medio Ambiente, 2001. Disponible en: https://agua. org.mx/biblioteca/justicia-ambiental-conostruccion-y-defensa-de-los-nuevos-derechos-ambientales-culturalesy-colectivos-en-america-latina-2/

MAASS, J. M. et al. Ecosystem services of tropical dry forests: insights from longterm ecological and social research on the Pacific Coast of Mexico. Ecology and society, v. 10, p. 1-23, 2005. Disponible en: https://www. ecologyandsociety.org/vol10/iss1/art17/ 
MARCH, H. Neoliberalismo y medio ambiente: una aproximación desde la geografía crítica. Documents d'anàlisigeogràfica, v. 59, p. 137-153, 2013.

MARTÍN-LÓPEZ,B.; GÓMEZ-BAGGETHUN, E.; GARCÍA-LLORENTE, M.; MONTES, C. Trade-offs across value-domains in ecosystem services assessment. Ecological Indicators, v. 37, p. 220-228, 2014.

MARTÍNEZ ALIER, J. La justicia ambiental y el decrecimiento económico: una alianza entre dos movimientos. Ecología política, v.41, p. 45-54, 2011. Disponible en: https://dialnet.unirioja.es/servlet/articulo?codigo=3720224

MARTÍNEZ ALIER, J. El Ecologismo de los pobres. 395 p. Barcelona, España:Editorial Icaria, 2009.

MCCAULEY, D. J. Selling out on nature. Nature,v. 443, p. 27-28, 2006.

MEA. Millennium Ecosystem Assessment: Ecosystems and Human Well-being, p. 3. Washington D.C.: Island Press, 2005.

MENZEL, S.; TENG, J. Ecosystem services as a stakeholder-driven concept for conservation science. Conservation Biology,v. 24, p. 907-909, 2010.

MONARES, A. Modernidad y crisis ambiental: en torno al fundamento de la relación naturaleza-ser humano en occidente. Revista Austral de Ciencias Sociales, v. 3, p. 31-42, 1999.

MOONEY, H. A.; EHRLICH, P. R., DAILY, G. E. Ecosystem services: a fragmentary history. In: Gaily, G. (Org). Nature's Services: societal dependence on natural ecosystems. Washington DC: Island Press, 1997.

NIETO-ROMERO, M.; OTEROS-ROZAS, E.; GONZÁLEZ, J. A.; MARTÍN-LÓPEZ, B. Exploring the knowledge landscape of ecosystem services assessments in Mediterranean agroecosystems: insights for future research. Environmental Science \& Policy,v. 37, p. 121-133, 2014.

NORGAARD, R. B. Ecosystem services: From eye-opening metaphor to complexity blinder. Ecological economics, v. 69, p.1219-1227, 2010.

OLTRA, C. Modernización ecológica y sociedad del riesgo: hacia un análisis de las relaciones entre ciencia, medio ambiente y sociedad. Papers: revista de sociologia, v. 78, p. 133-149, 2005. Disponible en: https://ddd.uab.cat/ pub/papers/02102862n78/02102862n78p133.pdf

QUETIER, F.; TAPELLA, E.; CONTI, G.; CÁCERES, D.; DÍAZ, S. Servicios ecosistémicos y actores sociales. Aspectos conceptuales y metodológicos para un estudio interdisciplinario. Gaceta ecológica, v. 84, p. 17-26, 2007. Disponible en: http://www.nucleodiversus.org/uploads/file/Papers/Quetier_etal_2007_GacetaEcologicaDeMexico.pdf

REYERS, B., ROUX, D. J., COWLING, R. M., GINSBURG, A. E., NEL, J. L., FARRELL, P. O. Conservation planning as a transdisciplinary process, Conservation Biology, v. 24, p. 957-965, 2010.

SANDBROOK, C.; ADAMS, W. M.; BUSCHER, B.; VIRA, B. Social research and biodiversity conservation. Conservation Biology,v. 27, p. 1487-1490, 2013.

SCHAICH, H.; BIELING, C.; PLIENINGER, T. Linking ecosystem services with cultural landscape research. GaiaEcological Perspectives for Science and Society, v. 19, p. 269-277, 2010.

SCHRÖTER, M.; ZANDEN, E. H.; OUDENHOVEN, A. P.; REMME, R. P.; SERNA-CHAVEZ, H. M.; GROOT, R. S.; OPDAM, $P$. Ecosystem services as a contested concept: a synthesis of critique and counter-arguments. Conservation Letters, v. 7, p. 514-523, 2014.

SEPPELT, R.; DORMANN, C. F.; EPPINK, F. V.; LAUTENBACH S., SCHMIDT, S. A quantitative review of ecosystem service studies: approaches, shortcomings and the road ahead. Journal of applied Ecology, v. 48, p. 630-636, 2011.

STENSEKE, M.; LARIGAUDERIE, A. The role, importance and challenges of social sciences and humanities in the work of the intergovernmental science-policy platform on biodiversity and ecosystem services (IPBES), Innovation. The European Journal of Social Science Research, v. 31, p. 10-14, 2018.

SULLIVAN, S. Banking nature? The spectacular financialisation of environmental conservation. Antipode, v. 45, p. 198-217, 2013. Disponible en: https://onlinelibrary.wiley.com/doi/full/10.1111/j.1467-8330.2012.00989.x 
SULLIVAN, S. Green capitalism, and the cultural poverty of constructing nature as service-provider. Radical Anthropology, v. 3, p. 18-27, 2009.

VATN, A. Rationality, institutions and environmental policy. Ecological Economics,v. 55, p. 203-217, 2005.

VATN, A. The environment as a commodity. Environmental Values,v. 9, p. 493-509, 2000.

VIHERVAARA, P.; RÖNKÄ, M.; WALLS, M. Trends in ecosystem service research: early steps and current drivers. Ambio,v. 39, p. 314-324, 2010.

WILSON, C. M.; MATTHEWS, W. H. Man's Impact on the Global Environment. Report of the Study of Critical Environmental Problems (SCEP), 1970. 